\title{
Combination of Linagliptin and Empagliflozin Preserves Cardiac Systolic Function in an Ischemia-Reperfusion Injury Mice With Diabetes Mellitus
}

\author{
Akihito Ideishi ${ }^{\text {a }}$ b, d, Yasunori Suematsu ${ }^{\text {a, d }}$, Kohei Tashiro ${ }^{a}$, Hidetaka Morita ${ }^{\text {a }}$, \\ Takashi Kuwano $^{\text {a }}$, Sayo Tomita ${ }^{\text {a }}$ Kanji Nakai ${ }^{\text {a }}$, Shin-ichiro Miura ${ }^{\text {a, c, e }}$
}

\begin{abstract}
Background: Sodium-glucose co-transporter 2 inhibitor (SGLT2i) and dipeptidyl peptidase 4 inhibitor (DPP4i) are oral hypoglycemic agents. Although SGLT2i has been shown having the beneficial effects on heart failure in basic and clinical studies, the combined effects of SGLT2 $i$ and DPP4i have not been established well. We investigated the effects of SGLT2i and DPP4i against diabetes mice model of myocardial ischemia-reperfusion injury.
\end{abstract}

Methods: Streptozotocin-induced diabetic C57BL/6J mice were divided into control (vehicle), empagliflozin ( $30 \mathrm{mg} / \mathrm{kg} / \mathrm{day})$, linagliptin $(3 \mathrm{mg} / \mathrm{kg} / \mathrm{day})$ and combination $(30 \mathrm{mg} / \mathrm{kg} /$ day and $3 \mathrm{mg} / \mathrm{kg} /$ day, respectively) groups. After 7 days of drug administration, 30 min of myocardial ischemia was performed. We investigated body weight, heart weight, blood glucose, and cardiac functions by pressure-volume Millar catheter followed by 28 days of additional drug administration.

Results: Blood glucose levels, body weight, and heart weight were not significantly different between the groups. In Millar catheter analysis, left ventricular volume at the peak left ventricular ejection rate which is one of the cardiac systolic parameters in combination group was significantly preserved than that in control $(P=0.036)$. The cardiac index in the combination group tended to be preserved compared to that in the control $(\mathrm{P}=0.06)$. The pathological fibrotic area in the left ventricle in the combination group also tended to be smaller $(\mathrm{P}=0.08)$.

Manuscript submitted November 4, 2020, accepted December 10, 2020

Published online January 24, 2021

aDepartment of Cardiology, Fukuoka University School of Medicine, 7-45-1, Nanakuma, Jonan-ku, Fukuoka, Japan

${ }^{b}$ Department of Cardiovascular Medicine, National Cerebral and Cardiovascular Center, 6-1 Kishibe-Shimmachi, Suita, Osaka, 564-8565, Japan

'Department of Cardiology, Fukuoka University Nishijin Hospital, Fukuoka, Japan

dThese authors were equally contributed as first authors.

${ }^{e}$ Corresponding Author: Shin-ichiro Miura, Fukuoka University School of

Medicine, 7-45-1 Nanakuma, Jonan-ku, Fukuoka 814-0180, Japan.

Email:miuras@cis.fukuoka-u.ac.jp

doi: https://doi.org/10.14740/cr1194
Conclusions: Combination therapy with linagliptin and empagliflozin preserved cardiac systolic function on the diabetes mice model of myocardial ischemia-reperfusion injury independent of blood glucose levels.

Keywords: Empagliflozin; Linagliptin; Cardiac systolic function; Myocardial reperfusion injury; Diabetes mellitus

\section{Introduction}

Sodium-glucose co-transporter 2 inhibitor (SGLT2i) is an oral hypoglycemic agent blocking reabsorbs of filtered glucose [1]. Sodium-glucose co-transporter 2 in the proximal tubule of the kidney reabsorbs $90 \%$ of filtered glucose and was augmented by hyperglycemia condition. SGLT2i prompts improving beta cell function and enhanced insulin sensitivity in liver and muscle [1]. Empagliflozin, which is one of SGLT2i, reduced primary composite cardiovascular outcome including death from cardiovascular causes, nonfatal myocardial infarction, or nonfatal stroke, and death from any cause with type 2 diabetes patients at high risk for cardiovascular events (EMPA-REG OUTCOME) [2]. The cardiovascular protective effect of empagliflozin has been expected an osmotic diuretic effects by promoting excretion of urine glucose [1], but many researchers suggest the other cardiovascular protective effects such as anti-fibrosis, anti-inflammation, improvement of mitochondrial function and endothelial dysfunction [3-8].

Dipeptidyl peptidase 4 inhibitor (DPP4i) is an oral antihyperglycemic drug which blocks degradation of incretins including glucagon-like peptide-1 and glucose-dependent insulinotropic polypeptide. Incretins are secreted by gastrointestinal tract into blood after food ingestion, and cause increased insulin secretion and decreased glucagon secretion by the pancreas [9]. Linagliptin, which is one of DPP4i, shows cardioprotective effects including anti-inflammation and antiremodeling in basic studies [10-13], and randomized, doubleblind, placebo-controlled clinical trial to explore the effect of linagliptin on left ventricular (LV) systolic function in type 2 diabetes patients in primary prevention regardless of glycemic control (DYDA $2^{\mathrm{TM}}$ trial) is ongoing [14]. 
Combination therapy with empagliflozin and linagliptin is effective for diabetes mellitus because the two drugs have different actions for diabetes mellitus $[15,16]$. The action of empagliflozin is removing glucose through the urine, and the action of linagliptin is increasing insulin secretion and decreasing the levels of glucagon in the circulation [15]. Genital infections associated with SGLT- $2 \mathrm{i}$ were reduced by added DPP4i, because of a better glucose control [17]. Besides the effect for diabetes mellitus, combination of empagliflozin and linagliptin improves hypertension and vascular function in type 2 diabetes [18], attenuates neointima formation after vascular injury in diabetic mice [19], and prevents steatohepatitis in mouse model of non-alcoholic steatohepatitis and diabetes [20]. However, the effect of combination therapy for myocardial ischemia-reperfusion injury with diabetes mellitus has not been established well. In this research, for the first time, we investigated the effect of empagliflozin and linagliptin on the mice model of myocardial ischemia-reperfusion injury with diabetes mellitus.

\section{Materials and Methods}

\section{Ethics}

All procedures conformed to the Guide for the Care and Use of Laboratory Animals of the Institute of Laboratory Animal Resources. All experimental protocols were approved by the Animal Care and Use Committee of Fukuoka University (\#1805007).

Male C57BL/6J mice were purchased from Japan SLC, Inc. (Japan). Mice were housed in a climate-controlled vivarium with $12 \mathrm{~h}$ day/night cycles with food and water ad libitum. After 1 week housing, 8 - 10 weeks old mice were infused 150 $\mathrm{mg} / \mathrm{kg}$ streptozotocin intraperitoneally [21]. We determined more than $300 \mathrm{mg} / \mathrm{dL}$ blood glucose level 7 days later as a diabetic mouse (day 0). Beginning the next day, diabetic mice were divided into control (vehicle), empagliflozin $(30 \mathrm{mg} / \mathrm{kg} /$ day), linagliptin (3 $\mathrm{mg} / \mathrm{kg} /$ day) and combination $(30 \mathrm{mg} / \mathrm{kg} /$ day and $3 \mathrm{mg} / \mathrm{kg} /$ day, respectively) groups and started drug administration by oral gavage. Empagliflozin and linagliptin were kindly provided by Boehringer Ingelheim International (Ingelheim, Germany). After 7 days drug administration, we performed 30-min myocardial ischemia as previously described [21] (day 7). Mice were anesthetized by inhalation of $1.5 \%$ isoflurane, and they were placed on a heating mat to maintain normothermia. Under the condition of positive pressure ventilation with a ventilator (CWE, Inc., Ardmore, PA, USA), the left anterior descending coronary artery was ligated at the edge level of the left atrium with silk 6-0 sutures tied transiently over PE10 tubing for $30 \mathrm{~min}$. Then the ligature was released and the chest was closed. Followed by 28 days additional drug administration, cardiac function was evaluated by a Millar catheter as previously described [21] (day 35). After thoracotomy with anesthesia, the Millar conductance catheter was inserted into the left ventricle from apical stab wound made by a 25 -gauge needle. While the measurement was performed, ventilation was paused.

\section{Blood glucose check}

Blood glucose level was checked with using Glutest Ai and Glutest Neo sensor (SANWA KAGAKU KENKYUSHO CO., LTD., Japan). We checked blood glucose level on day 0 for diagnosis of diabetes mellitus, day 14, and day 35 .

\section{Evaluation of cardiac function}

Millar conductance catheter (Integral 3model VPR-1003, Unique Medical Co., Ltd., Tokyo, Japan) was used for evaluation of LV function as previously described [21]. Maximum pressure, minimum pressure, maximum volume, minimum volume, endsystolic pressure, end-systolic volume, end-diastolic pressure, end-diastolic volume, LV ejection fraction, stroke work, arterial elastance, the maximum peak time derivative of LV pressure $(\mathrm{dP} /$ $\mathrm{dt}$ max), the minimum peak time derivative of LV pressure, LV diastolic time constant, and LV volume at the peak LV ejection rate $(\mathrm{dV} / \mathrm{dt} \mathrm{min})$ and at the peak LV filling rate were measured. Stroke index or cardiac index was calculated by stroke volume or cardiac output per body weight as previous reports [22, 23].

\section{Histological analysis}

The quantity of myocardial fibrosis was evaluated in Picrosirius red-stained heart sections as previously described [21]. LV tissue of mid-layer below the ligated area was fixed with $4 \%$ paraformaldehyde and stained by picrosirius red by Biopathology Institute Co. (Oita, Japan). For each section, digital photographs were taken and merged using a BZ-9000 series All-in-one Fluorescence Microscope (Keyence Japan, Osaka, Japan). The percentage of the fibrotic area in the left ventricle was analyzed using Image J software (v1.47).

\section{Quantitative reverse transcription-polymerase chain reac- tion (RT-PCR) analysis}

Gene expression levels were quantified by RT-PCR as previously described [21]. We investigated the apex area of left ventricle. RiboPure RNA Purification Kit (Life Technologies, Carlsbad, CA, AUSA) and ReverTra Ace ${ }^{\circledR}$ qPCR RT Kit (TOYOBO, Japan) were used for extracting total ribonucleic acid (RNA) and producing complementary deoxyribonucleic acid (cDNA), respectively. Quantitative RT-PCR was performed with a 7500 Fast Real-Time PCR System (Applied Biosystems) using a THUNDERBIRD ${ }^{\circledR}$ SYBR $^{\circledR}$ qPCR Mix (TOYOBO). The primers of transforming growth factor- $\beta$ (forward: AGCTGCGCTTGCAGAGATTA, reverse: ATTCCGTCTCCTTGGTTCAGC), interleukin-6 (forward: TGTGCAATGGCAATTCTGAT, reverse: GGTACTCCAGAAGACCAGAGGA), tumor necrosis factor- $\alpha$ (forward: AGCCCCCA GTCTGTATCCTT, reverse: GGTCACTGTCCCAGCATCT T), interleukin-1 $\beta$ (forward: CTCCAGCCAAGCTTCCTTGT, reverse: ACAGGTCATTCTCATCACTGTCAA), and $\beta$-actin (forward: GATCAAGATCATTGCTCCTCCTGA, reverse: AC 
Table 1. Blood Glucose Level and Heart Weight/Body Weight

\begin{tabular}{|c|c|c|c|c|}
\hline & Control $(n=6)$ & Linagliptin $(n=6)$ & Empagliflozin $(n=9)$ & Combination $(n=7)$ \\
\hline \multicolumn{5}{|l|}{ Blood glucose (mg/dL) } \\
\hline Day 0 & $397 \pm 104$ & $411 \pm 106$ & $441 \pm 107$ & $366 \pm 79$ \\
\hline Day 35 & $660 \pm 206$ & $549 \pm 180$ & $478 \pm 95^{\mathrm{a}}$ & $522 \pm 85$ \\
\hline Heart weight/body weight on day $35(\%)$ & $0.53 \pm 0.04$ & $0.50 \pm 0.03$ & $0.47 \pm 0.06$ & $0.48 \pm 0.07$ \\
\hline
\end{tabular}

${ }^{*} \mathrm{P}<0.05$, and ${ }^{\mathrm{P}} \mathrm{P}<0.10$ by one-way analysis of variance with post-hoc comparison using Dunnett's test.

GCAGCTCAGTAACAGTCC) were investigated.

\section{Statistical analysis}

The continuous variables are expressed as mean \pm standard deviation. Group differences were analyzed by one-way analysis of variance with post-hoc comparison using Dunnett's test. Paired observations were analyzed by paired $t$-test. Statistical significance was defined as a $\mathrm{P}$ value of $<0.05$.

\section{Results}

\section{Changes of blood glucose levels and body weight}

Changes of blood glucose levels and heart weight/body weight were shown in Table 1. Blood glucose levels at baseline and body weight were not significantly different between the groups. On day14, blood glucose levels in empagliflozin and combination groups were significantly lower than that in control group (empagliflozin vs. control, $\mathrm{P}=0.006$; and combination vs. control, $\mathrm{P}=0.005$ ). On day 35 , the differences of blood glucose levels between the groups were lost, although the difference of blood glucose levels between empagliflozin and control had a tendency $(\mathrm{P}=0.06)$. The changes of body weight in each group were not significantly different. On day 35 , the body weight, heart weight, and ratio of heart weight per body weight, which is one of cardiac hypertrophic parameters, were not significantly different compared to that in control group.

\section{Millar conductance catheter analysis}

LV systolic parameters were shown in Figure 1. DV/dt min
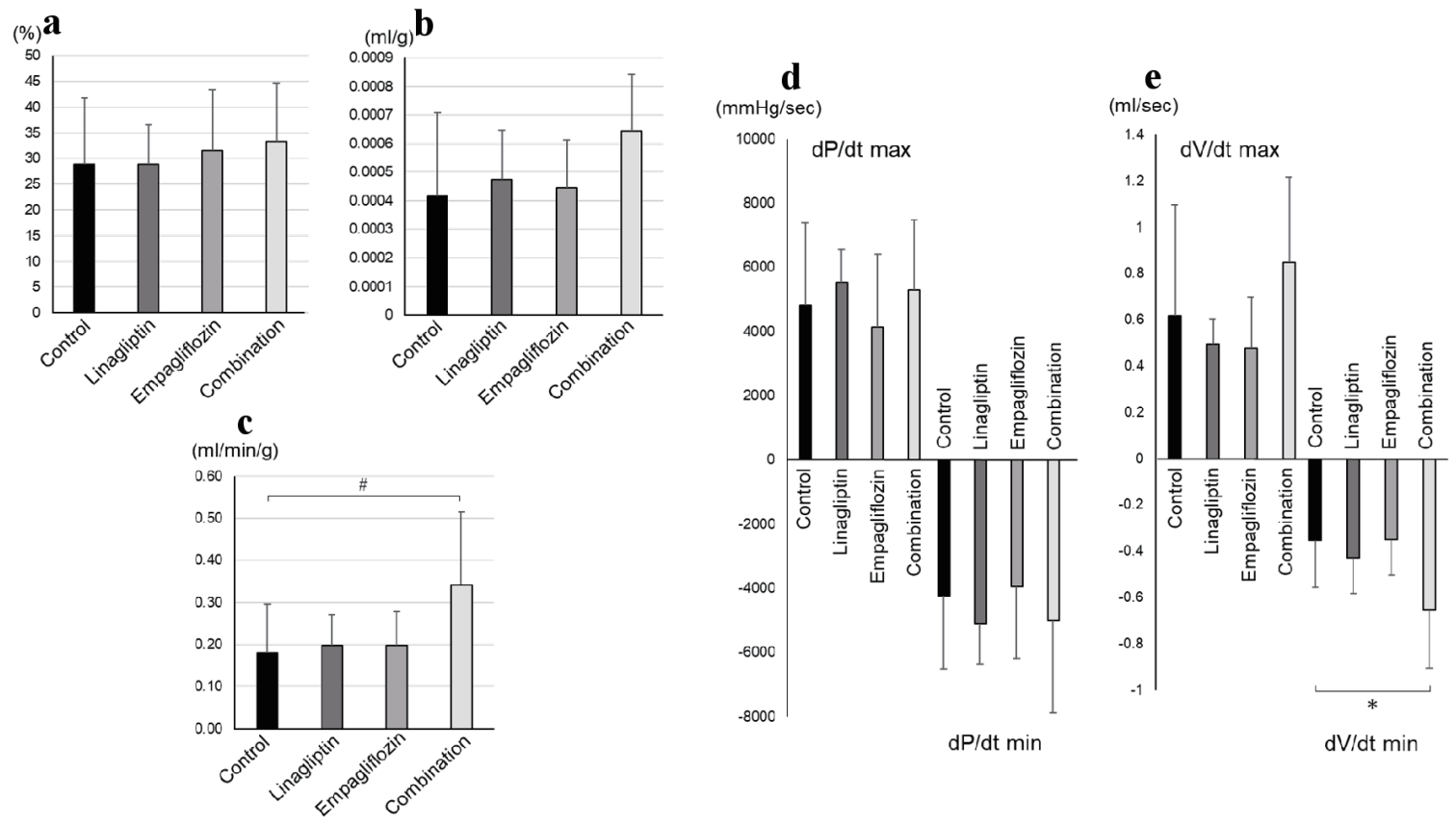

Figure 1. Cardiac systolic parameters by Millar conductance catheter: (a) LV ejection fraction, (b) stroke volume, (c) cardiac index, (d) dP/dt, and (e) dV/dt were shown. LV: left ventricular; dP/dt max: the maximum peak time derivative of LV pressure; $d P / d t$ min: the minimum peak time derivative of LV pressure; dV/dt max: LV volume at the peak LV filling rate; dV/dt min: LV volume at the peak LV ejection rate. The number of control, linagliptin, empagliflozin, and combination groups were $8,7,7$, and 6 , respectively. ${ }^{*} \mathrm{P}<0.05 ; \# \mathrm{P}<0.10$ by one-way analysis of variance with post-hoc comparison using Dunnett's test. 
Table 2. Millar Conductance Catheter Analyses Without LV Systolic Parameters

\begin{tabular}{lllll}
\hline & Control $(\mathbf{n}=\mathbf{8})$ & Linagliptin $(\mathbf{n}=\mathbf{7})$ & Empagliflozin $(\mathbf{n}=7)$ & Combination $(\mathbf{n}=\mathbf{6})$ \\
\hline P max $(\mathrm{mm} \mathrm{Hg})$ & $91 \pm 53$ & $81 \pm 8$ & $75 \pm 26$ & $98 \pm 38$ \\
P min (mm Hg) & $10 \pm 19$ & $6 \pm 6$ & $5 \pm 5$ & $9 \pm 4$ \\
V max (mL) & $0.038 \pm 0.009$ & $0.041 \pm 0.010$ & $0.035 \pm 0.009$ & $0.048 \pm 0.008$ \\
V min (mL) & $0.027 \pm 0.007$ & $0.029 \pm 0.007$ & $0.024 \pm 0.008$ & $0.032 \pm 0.009$ \\
ESP (mm Hg) & $85 \pm 54$ & $78 \pm 9$ & $69 \pm 27$ & $94 \pm 39$ \\
ESV (mL) & $0.028 \pm 0.008$ & $0.030 \pm 0.007$ & $0.024 \pm 0.008$ & $0.034 \pm 0.010$ \\
EDP (mm Hg) & $11 \pm 19$ & $8 \pm 6$ & $6 \pm 5$ & $10 \pm 5$ \\
EDV (mL) & $0.037 \pm 0.008$ & $0.040 \pm 0.008$ & $0.035 \pm 0.009$ & $0.047 \pm 0.008$ \\
HR (beats/min) & $449 \pm 44$ & $471 \pm 25$ & $429 \pm 41$ & $459 \pm 78$ \\
Ea (mm Hg/mL) & $9,156 \pm 5,541$ & $7,344 \pm 2,907$ & $7,831 \pm 5,494$ & $5,564 \pm 2,280$ \\
tau (ms) & $16 \pm 15$ & $10 \pm 3$ & $13 \pm 6$ & $11 \pm 6$ \\
\hline
\end{tabular}

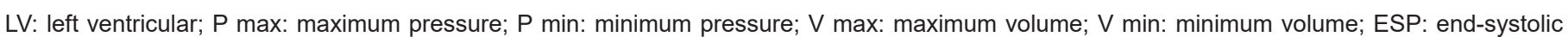

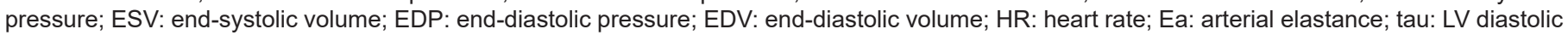
time constant.

in combination group was significantly preserved than that in control group (combination: $-0.65 \pm 0.25 \mathrm{~mL} / \mathrm{s}$, vs. control: $-0.35 \pm 0.20 \mathrm{~mL} / \mathrm{s}, \mathrm{P}=0.02)$. Cardiac index in combination group tended to be preserved than that in control group (combination: $0.34 \pm 0.17 \mathrm{~mL} / \mathrm{min} / \mathrm{g}$, vs. control: $0.18 \pm 0.12 \mathrm{~mL} /$ $\mathrm{min} / \mathrm{g}, \mathrm{P}=0.06$ ). On the other hand, the other LV systolic parameters including stroke index, $\mathrm{LV}$ ejection fraction, and $\mathrm{dP} /$ dt max were not significantly different between combination and control groups. Other parameters by Millar conductance catheter were also of no significant difference between the groups (Table 2).

The mRNA levels of various markers and anti-fibrotic effect of combination therapy with linagliptin and empagliflozin

The mRNA levels of various markers in left ventricle were analyzed (Supplementary Material 1, www. cardiologyres. org). The transforming growth factor- $\beta$ and inflammatory markers including interleukin-6, tumor necrosis factor- $\alpha$, and interleukin- $1 \beta$ were not significantly different between the groups. The results of pathological fibrotic analysis were shown in Figure 2. In picrosirius red staining, the ratio of fibrotic area per left ventricle in combination group tended to be smaller than that in control group (combination: $6.7 \pm 4.6 \%$, vs. control: $12.1 \pm 5.2 \%, \mathrm{P}=0.08)$.

\section{Discussion}

In this study, for the first time, we investigated the effects of combination therapy with linagliptin and empagliflozin against myocardial reperfusion injury with diabetes mellitus model mice. The combination group significantly preserved cardiac systolic function and showed a tendency of anti-fibrotic effect compared to control group independent of blood glucose levels.
The effect of combination therapy for myocardial ischemia-reperfusion injury with diabetes mellitus has not been reported. The mechanisms of the combination therapy for cardiovascular disease were unknown [24]. Myocardial reperfusion injury model is useful for clinical situation in this reperfusion era. We previously showed the effectiveness of angiotensin receptor neprilysin inhibitor against myocardial reperfusion injury with diabetes with using this model mouse [21]. We could show the improvement of $\mathrm{dV} / \mathrm{dt} \min$ by combination therapy, but not LV ejection fraction and $\mathrm{dP} / \mathrm{dt} \max$. DV/dt min is the peak of negative $\mathrm{dV} / \mathrm{dt}$ which indicates $\mathrm{LV}$ volume at $\mathrm{LV}$ ejection rate. Negative $\mathrm{dV} / \mathrm{dt}$ was indicated as one of LV systolic functions [25-27], and $\mathrm{dV} / \mathrm{dt}$ min occurred during the first third of systole [25]. Some reports were forcused on LV volume in early phase of LV systole for LV function [2830]. Johnson et al reported about LV volume ejected in early systole, and the volume of blood ejected in early systole was indicated as the ventricular performance [29]. The greater volume change in the first third of systolic ejection indicated the better ventricular performance. They discussed that a slow rate of volume change in early systolic ejection which is equivalent to reduction of $\mathrm{dV} / \mathrm{dt}$ min is characteristic of depresses ventricular performance. On the other hand, although positive $\mathrm{dV} / \mathrm{dt}$ which indicates LV volume at LV filling rate was also reported the valuable for LV function [31] and load-independent LV function [32]; our study could not show the significant improvement of $\mathrm{dV} / \mathrm{dt}$ max by combination therapy. As one of the reasons why combination therapy improved only $\mathrm{dV} / \mathrm{dt}$ min, $\mathrm{dV} / \mathrm{dt}$ min might be more sensitive LV systolic parameter than the other parameters. The $\mathrm{dV} / \mathrm{dt}$ min reflects LV mobility, because $\mathrm{dV} / \mathrm{dt}$ min includes not only the volume change, but also rate of volume change [33]. Second, $\mathrm{dV} / \mathrm{dt}$ min is strongly influenced by afterload. Combination therapy might reduce afterload, because SGLT2i has an osmotic diuretic effects by promoting excretion of urine glucose [1]. Since the reduction of afterload induces an increase in cardiac index, cardiac index tended to be increase in combination group. Since the $\mathrm{dV} / \mathrm{dt}$ 

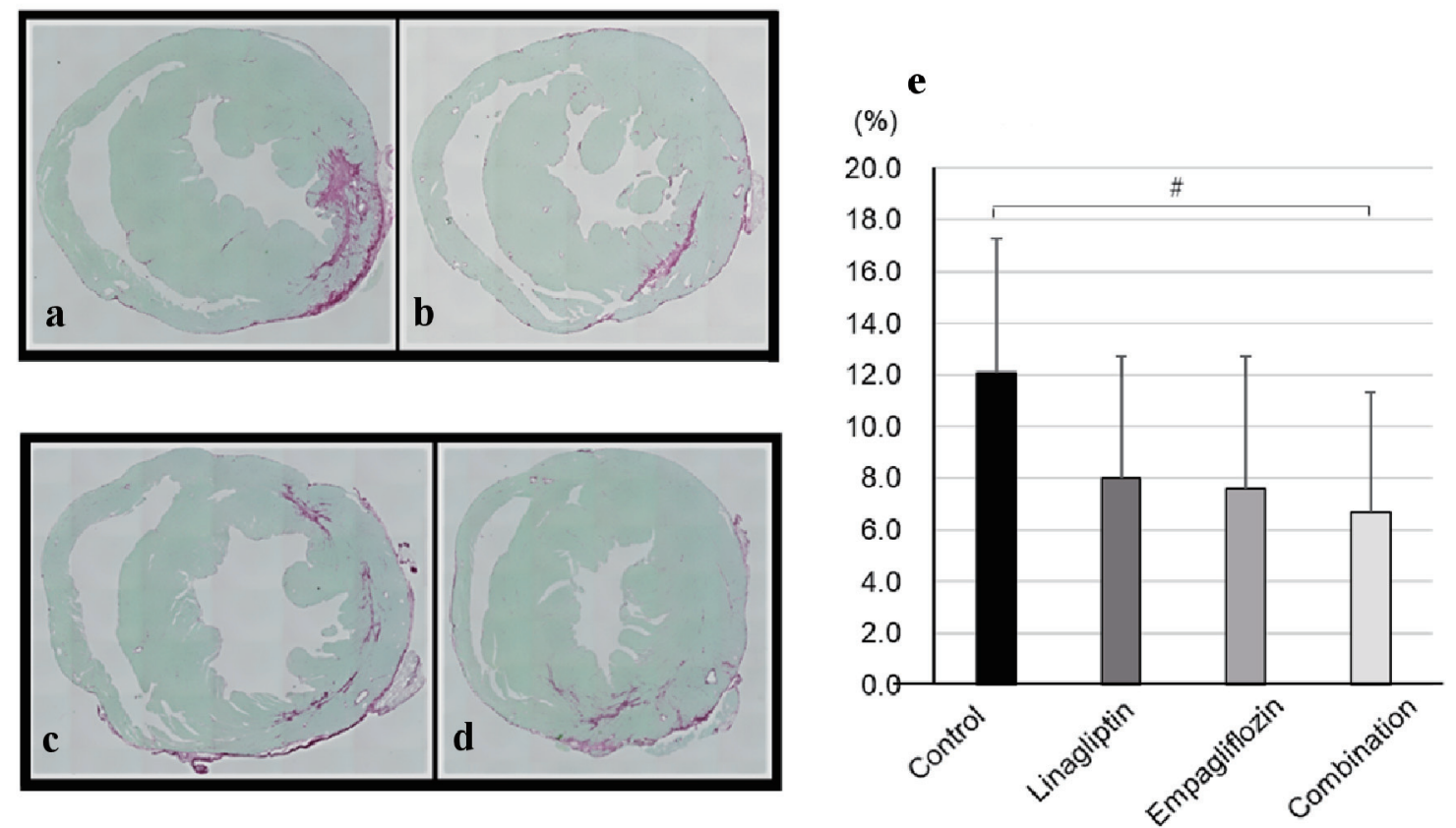

Figure 2. Representative photographs of picrosirius red-stained heart sections in (a) control, (b) linagliptin, (c) empagliflozin, and (d) combination groups were shown. (e) The ratios of fibrotic area per left ventricle were shown. The number of control, linagliptin, empagliflozin, and combination groups were $9,10,11$, and 8 , respectively. $\# \mathrm{P}<0.10$ by one-way analysis of variance with posthoc comparison using Dunnett's test.

min is one of established parameters of cardiac functions in the early systolic ejection, we might have been able to observe the significant reduction of $\mathrm{dV} / \mathrm{dt}$ min before the improvement of LV ejection fraction.

Combination therapy, but not monotherapy, induced the significant reduction of $\mathrm{dV} / \mathrm{dt}$ min. Although DPP4i improved cardiac function through various DPP-4 independent mechanisms including the attenuating oxidative stress [34] and stromal cell-derived factor-1 $\alpha$ [35], the effects of DPP4i were not enough for improvement of cardiac function in our experimental models. The diuretic effects of SGLT2 $i$ in addition to these effects of DPP4i might induce the beneficial effects of cardiac performance in this study.

The damage by streptozotocin-induced diabetes mellitus might be too strong in our models. Once, combination group significantly reduced blood glucose compared to control group on day 14, and after that the difference of blood glucose levels between the groups was lost on day 35. Although streptozotocin-induced diabetic mice were often used for type 2 diabetes mellitus research, it should be noted that the damage of streptozotocin would change the dose, duration [36] and strain [37]. A high single-dose streptozotocin causes rapid and massive $\beta$-cell necrosis leading to type- 1 diabetes [38]. Combination therapy might be able to show the effect of improvement of the other LV functional parameters under condition of lowdose streptozotocin or shortening experimental period.

\section{Conclusions}

The combination therapy with linagliptin and empagliflozin significantly preserved cardiac systolic function and showed a tendency of anti-fibrotic effect against myocardial reperfusion injury with diabetes model mice independent of blood glucose levels.

\section{Supplementary Material}

Suppl 1. Quantitative RT-PCR analysis.

\section{Acknowledgments}

None to declare.

\section{Financial Disclosure}

None to declare.

\section{Conflict of Interest}

S.M. has received grants and lecture honoraria from Boehringer Ingelheim.

\section{Informed Consent}

Not applicable. 


\section{Author Contributions}

Study concept and design: Akihito Ideishi, Yasunori Suematsu and Shin-ichiro Miura. Acquisition, analysis or interpretation of data: Akihito Ideishi and Yasunori Suematsu. Draft of the manuscript: Akihito Ideishi and Yasunori Suematsu. Critical revision of the manuscript for important intellectual content: Takashi Kuwano and Shin-ichiro Miura. Statistical analysis: Yasunori Suematsu. Administrative, technical, or material support: Kohei Tashiro, Hidetaka Morita, Sayo Tomita, and Kanji Nakai.

\section{Data Availability}

The authors declare that data supporting the findings of this study are available within the article.

\section{References}

1. Chawla G, Chaudhary KK. A complete review of empagliflozin: Most specific and potent SGLT2 inhibitor used for the treatment of type 2 diabetes mellitus. Diabetes Metab Syndr. 2019;13(3):2001-2008.

2. Zinman B, Wanner C, Lachin JM, Fitchett D, Bluhmki E, Hantel S, Mattheus M, et al. Empagliflozin, cardiovascular outcomes, and mortality in type 2 diabetes. N Engl J Med. 2015;373(22):2117-2128.

3. Connelly KA, Zhang Y, Desjardins JF, Nghiem L, Visram A, Batchu SN, Yerra VG, et al. Load-independent effects of empagliflozin contribute to improved cardiac function in experimental heart failure with reduced ejection fraction. Cardiovasc Diabetol. 2020;19(1):13.

4. Ganbaatar B, Fukuda D, Shinohara M, Yagi S, Kusunose K, Yamada H, Soeki T, et al. Empagliflozin ameliorates endothelial dysfunction and suppresses atherogenesis in diabetic apolipoprotein E-deficient mice. Eur J Pharmacol. 2020;875:173040.

5. Iannantuoni F, A MdM, Diaz-Morales N, Falcon R, Banuls C, Abad-Jimenez Z, Victor VM, et al. The SGLT2 inhibitor empagliflozin ameliorates the inflammatory profile in type 2 diabetic patients and promotes an antioxidant response in leukocytes. J Clin Med. 2019;8(11):1814.

6. Iborra-Egea O, Santiago-Vacas E, Yurista SR, Lupon J, Packer M, Heymans S, Zannad F, et al. Unraveling the molecular mechanism of action of empagliflozin in heart failure with reduced ejection fraction with or without diabetes. JACC Basic Transl Sci. 2019;4(7):831-840.

7. Shao Q, Meng L, Lee S, Tse G, Gong M, Zhang Z, Zhao $\mathrm{J}$, et al. Empagliflozin, a sodium glucose co-transporter-2 inhibitor, alleviates atrial remodeling and improves mitochondrial function in high-fat diet/streptozotocin-induced diabetic rats. Cardiovasc Diabetol. 2019;18(1):165.

8. Verma S, Sharma A, Zinman B, Ofstad AP, Fitchett D, Brueckmann M, Wanner C, et al. Empagliflozin reduces the risk of mortality and hospitalization for heart failure across thrombolysis in myocardial infarction risk score for heart failure in diabetes categories: Post hoc analysis of the EMPA-REG OUTCOME trial. Diabetes Obes Metab. 2020;22(7):1141-1150.

9. Aletti R, Cheng-Lai A. Linagliptin: the newest dipeptidyl peptidase-4 inhibitor for type 2 diabetes mellitus. Cardiol Rev. 2012;20(1):45-51.

10. Al Zoubi S, Chen J, Murphy C, Martin L, Chiazza F, Collotta D, Yaqoob MM, et al. Linagliptin attenuates the cardiac dysfunction associated with experimental sepsis in mice with pre-existing type 2 diabetes by inhibiting NF-kappaB. Front Immunol. 2018;9:2996.

11. Birnbaum Y, Tran D, Bajaj M, Ye Y. DPP-4 inhibition by linagliptin prevents cardiac dysfunction and inflammation by targeting the Nlrp3/ASC inflammasome. Basic Res Cardiol. 2019;114(5):35.

12. Gen WR, Fu CY, He HH, Zheng MZ, Wang LL, Yang Y, Shen YL, et al. Linagliptin improved myocardial function recovery in rat hearts after a prolonged hypothermic preservation. Life Sci. 2018;210:47-54.

13. Ishizue N, Niwano S, Niwano H, Oikawa J, Nakamura $\mathrm{H}$, Hashikata T, Igarashi $\mathrm{T}$, et al. Linagliptin suppresses electrical and structural remodeling in the isoproterenol induced myocardial injury model. Int Heart J. 2019;60(2):411-418

14. Giorda CB, Cioffi G, Lucci D, Nada E, Ognibeni F, Mancusi C, Latini R, et al. Effects of dipeptidyl peptidase-4 inhibitor linagliptin on left ventricular dysfunction in patients with type 2 diabetes and concentric left ventricular geometry (the DYDA 2 Trial). Rationale, design, and baseline characteristics of the study population. Cardiovasc Drugs Ther. 2019;33(5):547-555.

15. DeFronzo RA, Lewin A, Patel S, Liu D, Kaste R, Woerle HJ, Broedl UC. Combination of empagliflozin and linagliptin as second-line therapy in subjects with type 2 diabetes inadequately controlled on metformin. Diabetes Care. 2015;38(3):384-393.

16. Tan $X, H u$ J. Empagliflozin/Linagliptin: Combination therapy in patients with type 2 diabetes. Ann Endocrinol (Paris). 2016;77(5):557-562.

17. Fadini GP, Bonora BM, Mayur S, Rigato M, Avogaro A. Dipeptidyl peptidase- 4 inhibitors moderate the risk of genitourinary tract infections associated with sodiumglucose co-transporter-2 inhibitors. Diabetes Obes Metab. 2018;20(3):740-744.

18. Jung S, Bosch A, Kannenkeril D, Karg MV, Striepe K, Bramlage P, Ott C, et al. Combination of empagliflozin and linagliptin improves blood pressure and vascular function in type 2 diabetes. Eur Heart J Cardiovasc Pharmacother. 2020;6(6):364-371.

19. Takahashi H, Nomiyama T, Terawaki Y, Horikawa T, Kawanami T, Hamaguchi Y, Tanaka T, et al. Combined treatment with DPP-4 inhibitor linagliptin and SGLT2 inhibitor empagliflozin attenuates neointima formation after vascular injury in diabetic mice. Biochem Biophys Rep. 2019;18:100640.

20. Jojima T, Tomotsune T, Iijima T, Akimoto K, Suzuki K, Aso Y. Empagliflozin (an SGLT2 inhibitor), alone or in combination with linagliptin (a DPP-4 inhibitor), prevents steatohepatitis in a novel mouse model of non-alco- 
holic steatohepatitis and diabetes. Diabetol Metab Syndr. 2016;8:45.

21. Suematsu Y, Miura S, Goto M, Matsuo Y, Arimura T, Kuwano T, Imaizumi S, et al. LCZ696, an angiotensin receptor-neprilysin inhibitor, improves cardiac function with the attenuation of fibrosis in heart failure with reduced ejection fraction in streptozotocin-induced diabetic mice. Eur J Heart Fail. 2016;18(4):386-393.

22. Kawahara Y, Tanonaka K, Daicho T, Nawa M, Oikawa R, Nasa Y, Takeo S. Preferable anesthetic conditions for echocardiographic determination of murine cardiac function. J Pharmacol Sci. 2005;99(1):95-104.

23. Kreissl MC, Wu HM, Stout DB, Ladno W, Schindler TH, Zhang X, Prior JO, et al. Noninvasive measurement of cardiovascular function in mice with high-temporal-resolution small-animal PET. J Nucl Med. 2006;47(6):974980 .

24. Scheen AJ. Cardiovascular Effects of New Oral GlucoseLowering Agents: DPP-4 and SGLT-2 Inhibitors. Circ Res. 2018;122(10):1439-1459.

25. Bianco JA, Makey DG, Laskey WK, Shafer RB. Radionuclide left ventricular $\mathrm{dV} / \mathrm{dt}$ for the assessment of cardiac function in patients with coronary disease. J Nucl Med. 1979;20(1):1-6.

26. Soldo SJ, Norris SL, Gober JR, Haywood LJ, Colletti PM, Terk M. MRI-derived ventricular volume curves for the assessment of left ventricular function. Magn Reson Imaging. 1994;12(5):711-717.

27. Koito H, Spodick DH. Relation of left ventricular ejectional flow velocity to volume ejection rate. Am J Cardiol. 1989;63(3):256-257.

28. Hoshino T, Tomonaga G, Motomura M, Shimono Y, Kinoshita M, Kusukawa R. Quantitative analysis of left ventricular ejection phase by means of left ventricular cineangiography. Jpn Circ J. 1977;41(10):1085-1097.

29. Johnson LL, Ellis K, Schmidt D, Weiss MB, Cannon PJ. Volume ejected in early systole. A sensitive index of left ventricular performance in coronary artery disease. Circulation. 1975;52(3):378-389.
30. Marshall RC, Berger HJ, Costin JC, Freedman GS, Wolberg J, Cohen LS, Gottschalk A, et al. Assessment of cardiac performance with quantitative radionuclide angiocardiography: sequential left ventricular ejection fraction, normalized left ventricular ejection rate, and regional wall motion. Circulation. 1977;56(5):820-829.

31. Nakae I, Matsuo S, Koh T, Mitsunami K, Horie M. Left ventricular systolic/diastolic function evaluated by quantitative ECG-gated SPECT: comparison with echocardiography and plasma BNP analysis. Ann Nucl Med. 2005;19(6):447-454.

32. Kohli K, Kovacs SJ. The quest for load-independent left ventricular chamber properties: exploring the normalized pressure-volume loop. Physiol Rep. 2017;5(6):e13160.

33. Russell RO, Jr., Porter CM, Frimer M, Dodge HT. Left ventricular power in man. Am Heart J. 1971;81(6):799808.

34. Inthachai T, Lekawanvijit S, Kumfu S, Apaijai N, Pongkan W, Chattipakorn SC, Chattipakorn N. Dipeptidyl peptidase-4 inhibitor improves cardiac function by attenuating adverse cardiac remodelling in rats with chronic myocardial infarction. Exp Physiol. 2015;100(6):667679.

35. Connelly KA, Advani A, Zhang Y, Advani SL, Kabir G, Abadeh A, Desjardins JF, et al. Dipeptidyl peptidase-4 inhibition improves cardiac function in experimental myocardial infarction: Role of stromal cell-derived factor1alpha. J Diabetes. 2016;8(1):63-75.

36. Ito M. [The production of mouse model of slowly progressive diabetes mellitus and the preventive effect of low molecular weight chitosan on the progression of the diabetes mellitus]. Yakugaku Zasshi. 2013;133(7):773-782.

37. Hayashi K, Kojima R, Ito M. Strain differences in the diabetogenic activity of streptozotocin in mice. Biol Pharm Bull. 2006;29(6):1110-1119.

38. Kolb H. Mouse models of insulin dependent diabetes: low-dose streptozocin-induced diabetes and nonobese diabetic (NOD) mice. Diabetes Metab Rev. 1987;3(3):751778 . 